\title{
An IT System for the Remote Burden Optimization of Foundry Furnaces
}

\author{
Eugeniusz Ziółkowski \\ AGH University of Science and Technology, Faculty of Foundry Engineering, Reymonta 23 St., 30-059 Krakow, Poland \\ e-mail:ez@agh.edu.pl
}

(C) 2021 Author. This is an open access publication, which can be used, distributed and reproduced in any medium according to the Creative Commons CC-BY 4.0 License requiring that the original work has been properly cited.

Received: 25 June 2021 /Accepted: 21 July 2021/ Published online: 27 July 2021.

This article is published with open access at AGH University of Science and Technology Press

\begin{abstract}
The burden calculations for foundry furnaces are one of the most important steps in preparing the production of liquid casting alloys. These calculations, due to the usually large number of input materials and chemical elements, are realized by numerical methods. These methods are implemented in some spreadsheets, universal mathematical programs or in specialized programs for foundry engineering. The paper describes a computerized system for remote calculations of optimal burden. The technological, economic, and organizational features of implementing IT system have been presented, also taking into account its safety of use.
\end{abstract}

\section{Keywords:}

IT system for remote optimization, burden optimization for foundry furnaces

\section{INTRODUCTION}

Determining the optimal burden of an assumed chemical composition requires the formulation of an optimization task by defining an objective function (usually minimum charge cost) and a constraints system. Various mathematical models of such an optimization task have been described in publications including [1-4]. To solve such tasks, the following can be used:

- optimization module available in some spreadsheets (e.g. Solver in Excel/MS Office [5] or Solver in Calc/Libre-Office [6]);

- mathematical package (e.g., Mathematica by Wolfram Research [7], MATLAB by MathWorks [8]);

- specialized program ordered by foundries.

Using the optimization module contained in a spreadsheet as well as the optimal value search function in a mathematical package requires the ability to define a mathematical model. With the high dynamics of input data changes (changing the number and parameters of input materials, changing the list of chemical elements used in calculations), the use of these IT tools is inefficient. The much more effective realization of calculations in the given foundry is possible when a specialized program for burden optimization is implemented. However, it involves the need to purchase such a system, its constant servicing, updates, etc. Programs classified in this category can be implemented as single-user or multi-user programs, with one or more levels of user privileges to particular functions.
They can either be installed without any additional security or protected against unauthorized use and/or copying using, for example, dongles or network software licensing systems. The features of the above solutions have been the basis for the preparation of this paper presenting a universal computer system with remote burden optimization for foundry furnaces.

In the following part of the article the assumptions of this system and the way of its implementation will be described. The benefits of technologists using a remote calculation system in foundries will also be characterized.

\section{FUNCTIONAL ASSUMPTIONS OF THE IT SYSTEM FOR REMOTE BURDEN OPTIMIZATION}

The computation of the minimum cost batch bearing for the assumptions is to be performed by a server located at a selected computing center that authorized users can connect to via Internet connections. The developed system has the following features:

- preparation of input data for optimization calculations is performed by a program installed on a user's computer; this program is also used to obtain a connection to the calculation server, send the input data and receive the obtained calculation results from the server;

- the connection between a client and the server programs takes place over a computer network via the TCP/IP protocol on a specific port number; 
- calculations are performed by a program installed on the server; this program listens for connection requests from each client program and, without human intervention, after authentication and input validation, performs the calculations and then generates a report as an electronic document (in PDF format) and sends it to a client program from which the job was run;

- the program located on the server works continuously (24 hours a day) and is protected against unauthorized access and copying by means of a dongle on the USB port;

- there is also a database (PostgreSQL) installed on the server, containing data on the authorized users of the system along with additional information such as: the range of allowed calculation functions, the allowed number of calculation runs (if applicable), a date range in which a user can use the system and other organizational and possibly billing data;

- the program on the server runs under the control of the 64-bit MS Windows operating system;

- the program performs automatic cyclic backups of the database and reports from optimization calculations.

\section{MAIN PROGRAM INSTALLED ON THE SERVER}

The program installed on the server performs the following tasks:

- receives TCP network computation requests on a port with a specified number along with an XLSX format file (MS Excel);

- authorizes, in cooperation with database, the rights of a specific user to run a computational procedure;

- verifies the correctness of the input data;

- performs calculations using the selected fuzzy optimization method [2-4];

- generates a report of the calculations performed and sends a PDF file containing the input data and results to the user's computer;

- saves information about the actions performed in the database.

Figure 1 shows a view of the program window, developed and executed by the author of this publication, installed on a server named $R B C$ Server.

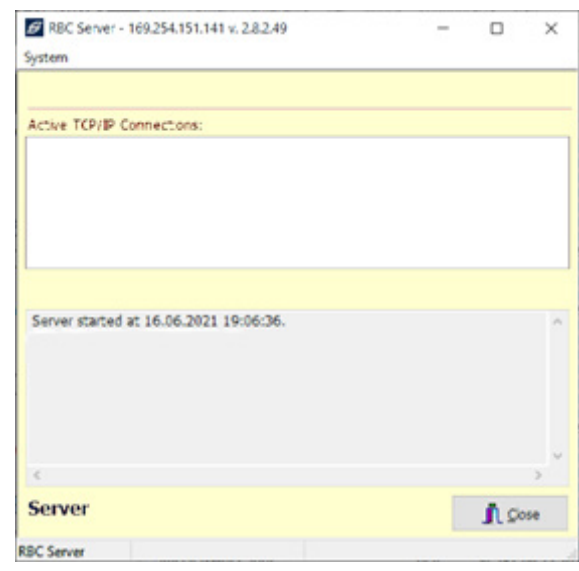

Fig. 1. View of RBC Server program window
This program was written in Embarcadero's RAD Studio 10.4.2 development environment [9]. DataSnap technology [10] is used to create networked multi-user applications that can listen for Internet requests from client programs and execute them. The program window displays only information about established connections and the status of successively performed actions. The $R B C$ Server program is protected against unauthorized use and copying by a Sentinel HL NetTime dongle [11] connected to the server's USB port. A view of such a key and its basic performance parameters are shown in Figure 2.

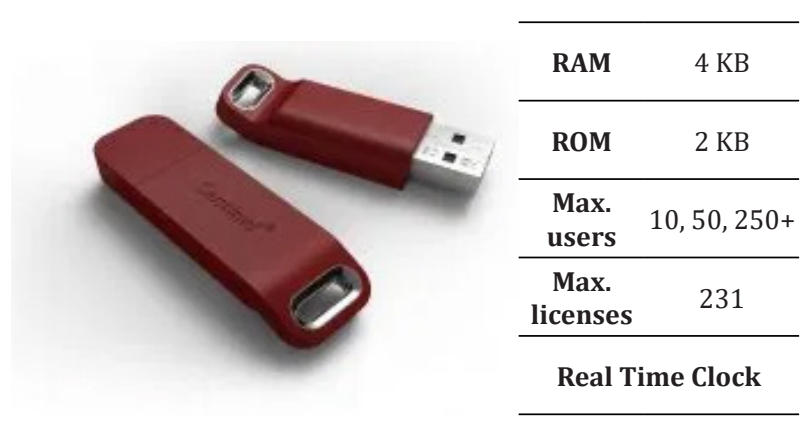

Fig. 2. View and basic parameters of the Sentinel HL NetTime dongle [11]

The Sentinel HASP Vendor Suite [12] is used to secure $R B C$ Server with a Sentinel HL NetTime dongle and to set and subsequently update the key's important operating parameters. Special Master and Developer dongles are also required to program this key. The HL NetTime key not only allows the specification of a number of RBC Server users, but also a license validity period which can be extended accordingly. This is especially important when installing the program on a server hosted by a third party. When a license expires, the program and the dongle can no longer be used.

\section{CUSTOMER PROGRAM}

The main tasks performed by the client program include:

- establishing a remote connection to RBC Server;

- authentication and reading the functional privileges from the server;

- creating a new, reading or modifying a stored input data file (in XLSX format);

- selection of a variant of optimization calculations of the burden for foundry furnace;

- sending the XLSX file to RBC Server;

- receiving a PDF file with the calculation results and displaying it on a client's computer in the default PDF viewer;

- the possibility to calculate the chemical composition of a charge for individually specified limits of particular input materials.

All communications between RBC Client and RBC Server are performed using SSL encryption. The connection and data exchange between RBC Server and the PostgreSQL database located on the server is also encrypted. 


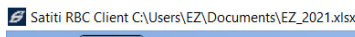

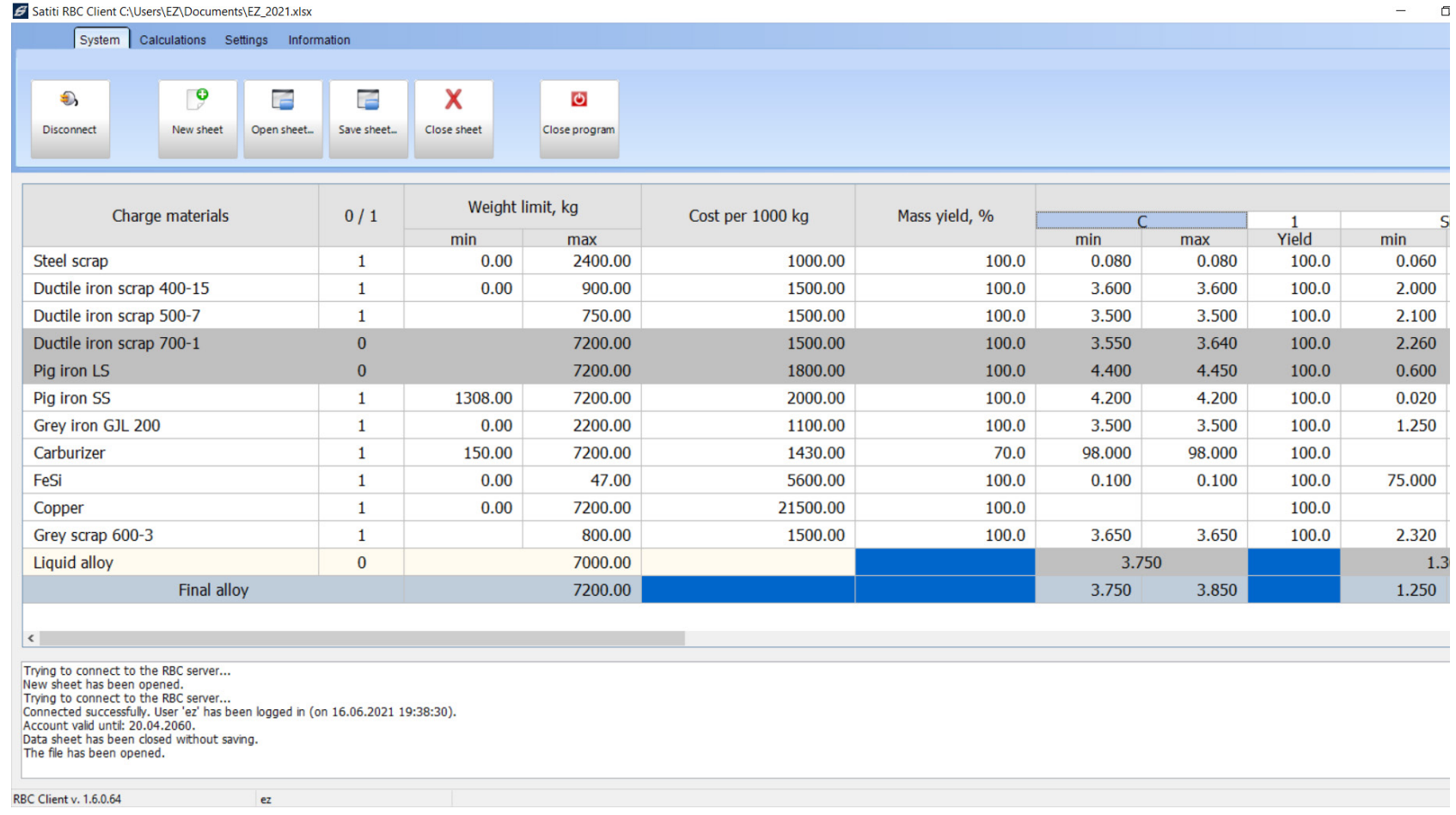

Fig. 3. View of RBC Client window with sample input data

Figure 3 shows a window view of the RBC Client with the sample input data, while a part of the PDF file with calculation results is shown in Figure 4.

Figure 5 shows a view of the main window of the client program ( $R B C$ Client) before establishing an authorized connection to RBC Server.

The input data for the optimal burden calculation includes the following information:

- names of change materials;

- in the ' $0 / 1$ ' column whether the input material should be considered for the calculation or not;

- the limit on the mass contribution of a given component to the input [kg];

- unit cost of each charge material;

- the chemical composition of each material, i.e. the range of each chemical element content to be considered in the calculation [\%];

- metallurgical yield value [\%];

- yield values of individual chemical elements [\%];

- the mass $[\mathrm{kg}]$ and chemical composition [\%] of the liquid metal from the previous melt;

- the requested values of the mass [kg] and chemical composition [\%] of the liquid melt after melting.

Once the connection to RBC Server has been established and the user has been properly authenticated, it is possible to select the optimization calculation mode in the RBC Client. It is now possible to determine the optimal burden (with minimum cost) and to calculate the minimum mass of charge to correct the incorrect chemical composition of the liquid melt in the foundry furnace (Fig. 4).

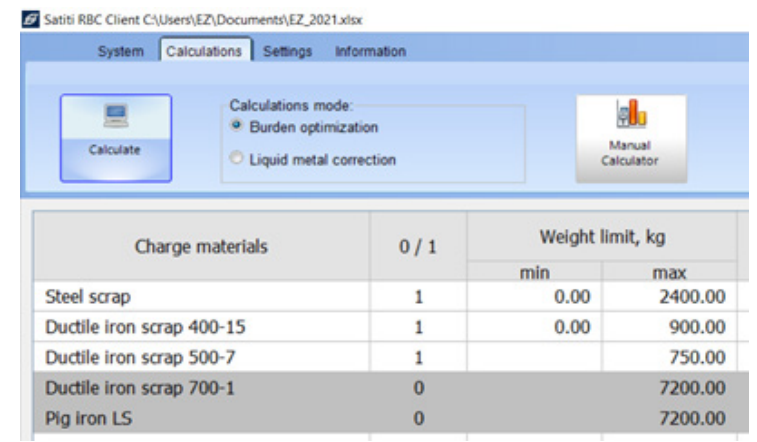

Fig. 4. Fragment of a window with selection of optimization calculation mode

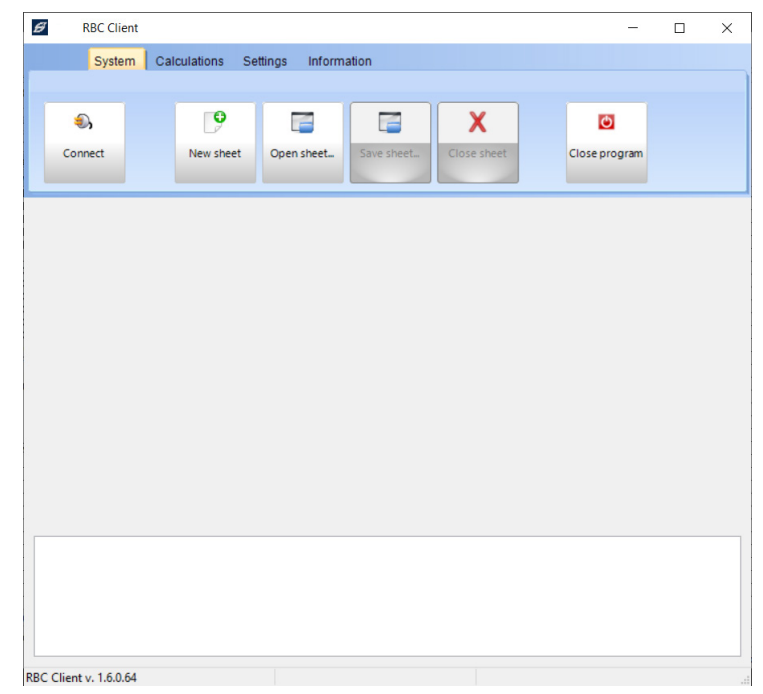

Fig. 5. View of RBC Client main window before logging on to RBC Server 
After the RBC Server program performs the calculations, a PDF file is automatically sent to a client computer, a part of which is shown in Figure 6. This file can be opened by the default PDF viewer and can be saved or printed by a customer.

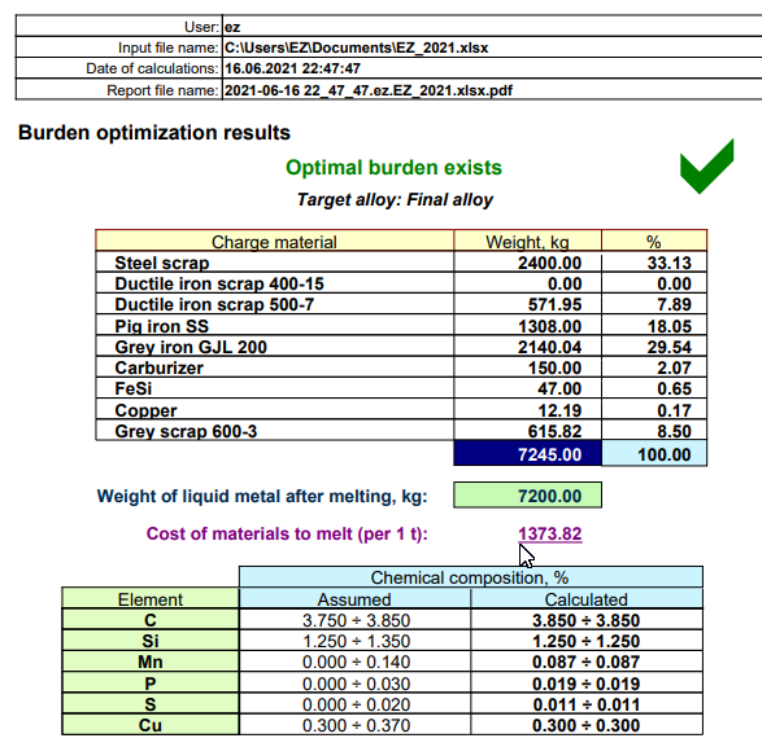

Fig. 6. Excerpt from the report (PDF file) with calculation results

\section{SUMMARY}

The application of tools for creating client-server applications (DataSnap technology in RAD Studio environment) with PostgreSQL database and implemented methods of burden optimization made it possible to create an effective remote IT system for determining charges for foundry furnaces. The application security used (SSL protocol, network and time hardware key protection) also allows the system to be used for commercial purposes. The created and practically tested IT system confirmed the easy management of users' authorizations, high efficiency of calculations' realization and advantages resulting from the possibility of automatic calculations' realization in any time. This allows it to be used regardless of where users' computers are located in the global computer network.

The developed system can also be used for other computational tasks.

\section{REFERENCES}

[1] Ziółkowski E. (2007). Algorithms of furnace charge burden optimisation in foundries. Archives of Metallurgy and Materials, 52(3), 487-495.

[2] Ziółkowski E. (2013). Algorithm for burden calculation for foundry furnaces using charge materials with an uncertain composition. Archives of Metallurgy and Materials, 58(3), 887-889.

[3] Ziółkowski E. (2017). Burden optimisation of lump charge materials for foundry furnaces. Archives of Metallurgy and Materials, 62(4), 2217-2221. Doi: https://doi.org/10.1515/ amm-2017-0327.

[4] Ziółkowski E. \& Schmalenberg K. (2019). Determination of the charge materials range in multistage charge burden optimization for foundry furnaces. Journal of Materials Engineering and Performance, 28(7), 4012-4017. Doi: https://doi.org/10.1007/ s11665-019-04144-6.

[5] Define and solve a problem by using Solver. Retrieved from: https:// support.microsoft.com/en-us/office/define-and-solve-a-problem-by-using-solver-5d1a388f-079d-43ac-a7eb-f63e45925040 [21.03.2021].

[6] Solver. LibreOffice 6.1 Help. Retrieved from: https://help.libreoffice.org/6.1/en-US/text/scalc/01/solver.html [18.05.2021].

[7] Wolfram Mathematica. Retrieved from: https://www.wolfram.com [21.03.2021].

[8] MATLAB Capabilities. Retrieved from: https://nl.mathworks. com/products/matlab.html [10.02.2021].

[9] RAD Studio 10.4.2. Retrieved from: https://www.embarcadero. com/products/rad-studio [7.04.2021].

[10] DataSnap Overview and Architecture. Retrieved from: http:// docwiki.embarcadero.com/RADStudio/Sydney/en/DataSnap_ Overview_and_Architecture [11.03.2021].

[11] Sentinel HASP. Award-winning, out-of-the-box Software Licensing Security Solution. Retrieved from: https://cpl.thalesgroup.com/software-monetization/all-products/sentinel-hasp [19.02.2021].

[12] Major Components of the Vendor Suite. Retrieved from: https:// docs.sentinel.thalesgroup.com/ldk/LDKdocs/SPNL/LDK_ SLnP_Guide/Familiarizing/Vendor_suite_components.htm [26.04.2021]. 